\author{
K. Montet-Abou \\ J. L. Daire \\ M. K. Ivancevic \\ J. N. Hyacinthe \\ D. Nguyen \\ M. Jorge-Costa \\ D. R. Morel \\ J. P. Vallée
}

\section{Optimization of cardiac cine in the rat on a clinical 1.5-T MR system}

Received: 23 December 2005

Revised: 9 June 2006

Accepted: 12 June 2006

Published online: 25 July 2006

(C) ESMRMB 2006
K. Montet-Abou ( $\varangle)$. J. L. Daire

M. K. Ivancevic - J. N. Hyacinthe

D. Nguyen · J. P. Vallée

Radiology and Medical Informatics

Department, Digital Imaging Unit (UIN),

Geneva University Hospital,

1211, Geneva 14,

Switzerland

E-mail: Karin.Montet-Abou@hcuge.ch

Tel.: +41-22-3795784

Fax: +41-22-3727072

M. Jorge-Costa - D. R. Morel

Anesthesiology, Pharmacology and

Intensive Care in Surgery Department,

Anesthesiological Investigations Unit,

Geneva University Hospital,

1211, Geneva 14

Switzerland

\author{
Abstract Object: the overall goal \\ was to study cardiovascular function \\ in small animals using a clinical \\ 1.5-T MR scanner optimizing a fast \\ gradient-echo cine sequence to \\ obtain high spatial and temporal \\ resolution. \\ Materials and methods: normal rat \\ hearts $(n=9)$ were imaged using a \\ 1.5-T MR scanner with a spiral fast \\ gradient-echo (fast field echo for \\ Philips scanners) sequence, three \\ Cartesian fast gradient-echo (turbo \\ field echo for Philips scanners) \\ sequences with different in-plane \\ resolution, and with and without \\ flow compensation and half-Fourier \\ acquisition. The hearts of four rats \\ were then excised and left-ventricle \\ mass was weighed. Inter- and \\ intra-observer variability analysis \\ was performed for magnetic \\ resonance imaging (MRI) \\ measurements.
}

Results: half-Fourier acquisition with flow compensation gave the best sequence in terms of image quality, spatial as well as temporal resolution, and suppression of flow artifact. Ejection fraction was $71 \pm$ $4 \%$ with less than $5 \%$ inter- and intra-observer variability. A good correlation was found between MRI-calculated left-ventricular mass and wet weight.

Conclusions: using optimized sequences on a clinical 1.5-T MR scanner can provide accurate quantification of cardiac function in small animals and can promote cardiovascular research on small animals at $1.5-\mathrm{T}$.

Keywords MRI - Cardiac imaging . Small animal

\section{Introduction}

Cardiac magnetic resonance imaging (MRI) already has well-established clinical applications with a constantly increasing list of indications [1-5]. In addition to clinical use, there is intense research into imaging and assessing the heart function of small animals to better understand cardiovascular pathologies. Some investigators have demonstrated the feasibility of imaging and quantifying the heart function and dysfunction using 2-T [6,7], 4.7-T [8-11], or higher-field MR systems [1214], dedicated exclusively to the small animal. However, such high-field systems are not widely available in contrast to 1.5-T MR systems used for clinical and research applications. Few animal studies at 1.5-T have evaluated the cardiac function of mice or rats [15-18], and have been limited by the spatial or temporal resolution and image quality. In particular, the optimization made possible by a clinical 1.5-T MR system has not been explored in these preliminary studies. Therefore, cardiac imaging of rodents has not been completely developed on clinical 1.5-T MR systems and remains a technical challenge due to the small size of the animals, fast heart and breathing rates, as well as the lower signal-to-noise ratio (SNR) of low-field MR systems. 
The aim of this work was: (1) to demonstrate that cardiac imaging of rats is feasible at 1.5-T on a clinical MR scanner with high spatial and temporal resolution (close to that achieved in humans), (2) to compare four different optimized fast gradient-echo cine sequences in the evaluation of heart function.

\section{Materials and methods}

\section{Animal preparation}

Anesthesia was induced in nine adult male rats (Sprague-Dawley) weighing $381 \pm 18 \mathrm{~g}$ with isoflurane $3 \%$, and was maintained by an intraperitoneal (IP) catheter with a bolus injection of $40 \mathrm{mg} / \mathrm{kg}$ sodium pentobarbital (Nembutal sodium solution, Abbott Laboratories, North Chicago, IL, USA) followed by continuous perfusion $(20 \mathrm{mg} / \mathrm{kg} / \mathrm{h})$. The rats were then placed in a cradle in supine position. A heating system maintained a constant temperature of the rats $\left(37-37.5^{\circ} \mathrm{C}\right)$, monitored by a rectal thermal probe; a respiratory pillow was placed on the abdomen to control the breathing rate. The electrocardiogram (ECG) recorded by subdermal electrodes was then used as a cardiac trigger for the MR system. Monitoring devices were connected with optical fibers to a personal computer (PC, Dell Latitude 800 with Windows 2000) during the MRI acquisitions [SA Instrument, Inc. (SAII), Stony Brook, NY, USA, MR-compatible model 1025 monitoring and gating system]. The data-acquisition modules were located near the animal in the MR scanner; a control/gating module was connected to a PC next to the operator console. Modules were controlled by menu-driven software from the PC. All output from ECG/temperature, respiration/IBP (pressure transducer) was transmitted through optical fibers. This system allowed acquisitions with ECG-gated sequences despite the high heart rate of the rat; furthermore, it allowed for continuous real-time physiological parameters assessment during the whole experiment. All animal procedures were approved by the ethical committee of our institution.

\section{Ex-vivo measurements}

Four rats were killed directly after the imaging session. The heart was carefully dissected to isolate the left ventricle, which was then weighed on an analytical balance (Acculab, Sartorius Group, Epsom, UK).

\section{MRI experiments}

Experiments were performed on a 1.5-T MR system (Intera, Philips Medical Systems, Best, The Netherlands) using a microscopy surface coil (47 mm diameter) for reception. A body coil was used for transmission. Four standard Philips MR fast gradient-echo (fast field echo on Philips system) sequences were used to acquire one long-axis and two short-axis views of the heart (mid-ventricular and apical): (1) spiral gradient echo [spiral fast field echo (FFE) sequence on Philips system], (2) Cartesian gradient-echo sequence with short repetition time (TR) and echo time (TE) [turbo field echo (TFE) sequences on clinical Philips scanner] with a high in-plane resolution (TFE-highres), (3) Cartesian gradient-echo sequence with short TR and TE with a low in-plane resolution (TFE-lowres), (4) a Cartesian TFE sequence with flow compensation and with half-Fourier acquisition (TFEhighres-flowcomp). TFE are gradient echo sequences with very short TE and TR which acquire $k$-space data while approaching steady state. In addition, contiguous short slices covering the entire ventricle length were acquired with the TFE-highres-flowcomp sequence for the four rats whose hearts were excised and weighted just after the MRI session.

All the sequences were synchronized to the ECG but no respiratory triggering was used. The animal-specific gating system allowed adaptations of clinical sequences for very high heart rates. Parameters of each sequence are detailed in Table 1.

\section{Image analysis}

Image analysis was performed on PowerMac G5 (Apple) using the open-source software OsiriX (http://homepage.mac. com/rossetantoine/osirix/HomePage.html).

Signal-to-noise ratio was measured in the left-ventricle blood, in the interventricular septum (myocardium), and in the muscles of the anterior thoracic wall. Signal intensity (SI) for each structure was averaged inside a manually drawn region of interest (ROI) of at least 20 voxels and then divided by the standard deviation (SD) of the background noise.

Blood-myocardium contrast-to-noise ratio (CNR) was eval-uated according to $\mathrm{CNR}=[\mathrm{SI}($ blood $)-\mathrm{SI}$ (myocardium) $] /$ $\mathrm{SD}$ (noise). To compare the different sequences, SNR and CNR values were normalized by the voxel size.

For each animal, ejection fraction (EF) was assessed as:

Ejection fraction $(\%)=\left(V_{\text {diast }}-V_{\text {syst }}\right) / V_{\text {diast }}$.

End-diastolic ( $\left.V_{\text {diast }}\right)$ and end-systolic $\left(V_{\text {syst }}\right)$ volumes were calculated by manually drawing endocardial contours and longaxis length at end-diastolic and end-systolic phases for each axis. End-diastolic and end-systolic volumes were calculated using the Simpson's modified method according to:

Volume $=($ length $/ 2)\left(A_{\text {mitral }}+(2 / 3) A_{\text {apex }}\right)$

where $A_{\text {mitral }}$ is the diastolic/systolic mitral valve area; $A_{\text {apex }}$ is the diastolic/systolic area, and length is the diastolic/systolic long-axis length.

Additionally, volumes were calculated by measuring the surface differences between blood and myocardium for each contiguous slice covering the entire ventricle length. Then the surfaces were multiplied by the slice thickness $(2 \mathrm{~mm})$ and summed.

Left-ventricle mass (LVM) was calculated according to the product of difference between the left-ventricle blood volume and left-ventricle volume (blood + myocardium) by the volumic mass $(1.05 \mu \mathrm{g} / \mu \mathrm{l})$ of the rat heart. 
Table 1 Comparison and summary of parameters for sequence acquisition, as well as the rat heart rate

\begin{tabular}{|c|c|c|c|c|}
\hline & Spiral-FFE & $\begin{array}{l}\text { TFE-highres- } \\
\text { flowcomp }\end{array}$ & TFE-highres & $\begin{array}{l}\text { TFE- } \\
\text { lowres }\end{array}$ \\
\hline Heart rate & 360 & 360 & 360 & 360 \\
\hline Acquisition time & 1'26 & 1’26 & 1'16 & 1’04 \\
\hline FOV (mm) & 120 & 160 & 160 & 160 \\
\hline RFOV (\%) & - & 60 & 60 & 60 \\
\hline Matrix scan & 256 & 288 & 288 & 160 \\
\hline $\begin{array}{l}\text { Matrix } \\
\text { reconstruction }\end{array}$ & 512 & 512 & 512 & 256 \\
\hline $\begin{array}{l}\text { Measured } \\
\text { voxel size }(\mathrm{mm})\end{array}$ & $0.47 / 0.47 / 2.0$ & $0.56 / 0.56 / 2.0$ & $0.56 / 0.56 / 2.0$ & $1.0 / 1.0 / 2.0$ \\
\hline $\begin{array}{l}\text { Reconstructed } \\
\text { voxel size }(\mathrm{mm})\end{array}$ & $0.23 / 0.23 / 2.0$ & $0.31 / 0.31 / 2.0$ & $0.31 / 0.31 / 2.0$ & $0.62 / 0.62 / 2.0$ \\
\hline Technique & FFE & TFE & TFE & TFE \\
\hline Flip angle (deg) & 30 & 15 & 15 & 15 \\
\hline NSA & 1 & 6 & 4 & 6 \\
\hline $\mathrm{TR}(\mathrm{ms})$ & 12.9 & 7.7 & 9.6 & 5.5 \\
\hline $\mathrm{TE}(\mathrm{ms})$ & 3.7 & 4.1 & 3.5 & 2.7 \\
\hline $\begin{array}{l}\text { Number of } \\
\text { phases/cycle }\end{array}$ & 12 & 14 & 11 & 20 \\
\hline RR window $(\%)$ & 50,15 & 10,15 & 10,20 & 10,15 \\
\hline $\begin{array}{l}\text { Cardiac } \\
\text { synchronization }\end{array}$ & Prospective & Retrospective & Retrospective & Retrospective \\
\hline $\begin{array}{l}\text { Flow } \\
\text { compensation }\end{array}$ & - & Yes & No & No \\
\hline $\begin{array}{l}\text { Half-Fourier } \\
\text { acquisition }\end{array}$ & No & Yes & No & No \\
\hline Partial echo & No & Yes & No & No \\
\hline $\begin{array}{l}\text { Partial echo } \\
\text { factor }\end{array}$ & - & 0.6 & - & - \\
\hline $\begin{array}{l}\text { Bandwidth } \\
\text { per pixel }(\mathrm{Hz})\end{array}$ & - & 496.7 & 496.7 & 723.8 \\
\hline $\begin{array}{l}\text { Water fat shift } \\
\text { (pixel) }\end{array}$ & - & 0.437 & 0.437 & 0.300 \\
\hline TFE SHOTs & - & 43 & 57 & 32 \\
\hline TFE FACTOR & - & 3 & 3 & 3 \\
\hline $\begin{array}{c}\text { Interleaves } \\
\text { number }\end{array}$ & 256 & & & \\
\hline
\end{tabular}

The acquisition time is given for a single slice. $F O V$ field of view; $R F O V$ rectangular field of view; $N S A$ number of signal averaging; $R R$ window time delay between two R waves of the ECG; TFE SHOTS number of shots in case of multishot acquisition to acquire all the $k$-space, depending on the TFE factor; TFE FACTOR percentage of matrix acquired within one TR, defines consequently the number of shots to acquire all the $k$-space; FFE fast field echo TFE turbo field echo flowcomp flow compensation; highres high resolution; lowres low resolution

\section{Image quality}

All images (all frames) were observed in a consensus reading by three investigators in order to score qualitatively image quality obtained from each sequence. The following criteria were used: presence of flow artifact in the ventricles, blood-myocardium edge sharpness, and signal homogeneity of myocardium. All images were qualitatively assessed on a five points scale [from minus minus (worst) to plus plus (best), passing through zero].
Reproducibility and variability analysis

Each set of data obtained with the TFE-highres-flowcomp sequence was analyzed by three different investigators in triplicate to evaluate inter- and intra-observer variability. Each investigator traced by hand the endocardial and epicardial contours for each end-diastolic and end-systolic slice. The left-ventricle length was also manually traced at end-diastole and end-systole. The areas (in $\mathrm{cm}^{2}$ ) and lengths (in $\mathrm{cm}$ ) were then gathered and compared statistically. 
Fig. 1 Representative example of images obtained with turbo field echo (TFE) sequence with flow compensation and half-Fourier acquisition (TFE-highres-flowcomp) for a complete cardiac cycle. The myocardium has a homogeneous signal during the whole contraction cycle in short axis at mitral (a) and apex (b) level and in long axis (c) and is also well differentiated from the blood. Flowcomp flow compensation; highres high resolution. Scale bar $=1 \mathrm{~cm}$
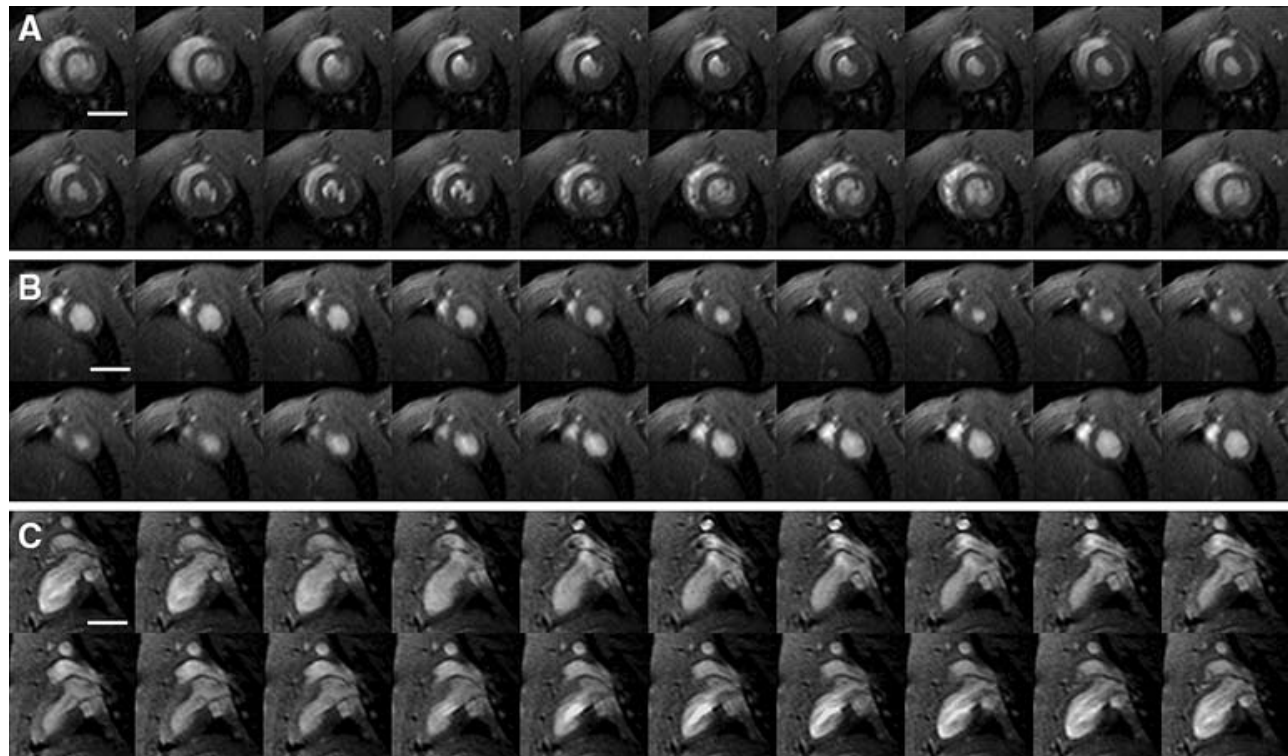

A one-way analysis of variance was performed for each measurement. A $p$ value inferior to 0.05 was considered significant.

\section{Results}

The cine protocols were successfully obtained in all the rats even at high heart rate ( 371 beats per minute (bpm) $\pm 57 \mathrm{bpm})$. An example of the image quality of the cine MR sequences is presented in Fig. 1. A high contrast between the blood and the myocardium was observed in all the series. SNR and CNR values for the left-ventricle blood and myocardium are presented in Table 2. The results of the consensus reading are presented in Table 3 . The flow compensation with complex-conjugate reconstruction (half-Fourier acquisition) provided a homoge-

Table 2 Signal-to-noise ratio (SNR) for blood of left ventricle and myocardium and contrast-to-noise ratio (CNR) between blood of left ventricle and myocardium per voxel volume unit (mean \pm SD)

\begin{tabular}{|c|c|c|c|c|}
\hline & Spiral-FFE & $\begin{array}{l}\text { TFE-highres- } \\
\text { flowcomp }\end{array}$ & $\begin{array}{l}\text { TFE- } \\
\text { highres }\end{array}$ & $\begin{array}{l}\text { TFE- } \\
\text { lowres }\end{array}$ \\
\hline SNR blood LV & $367 \pm 52$ & $265 \pm 35$ & $265 \pm 40$ & $307 \pm 78$ \\
\hline $\begin{array}{l}\text { SNR } \\
\text { myocardium }\end{array}$ & $153 \pm 28$ & $133 \pm 19$ & $153 \pm 26$ & $135 \pm 37$ \\
\hline $\begin{array}{l}\text { CNR blood } \\
\text { myocardium }\end{array}$ & $214 \pm 48$ & $133 \pm 40$ & $112 \pm 55$ & $173 \pm 43$ \\
\hline
\end{tabular}

Only the spiral-FFE sequence shows a statistical difference for the parameters SNR blood LV $(p<0.007)$ and CNR blood myocardium $(p<0.015)$ compared to the TFE-flowcomp and the TFE sequences. FFE fast field echo; TFE turbo field echo, flowcomp flow compensation, highres high resolution; lowres low resolution
Table 3 Qualitative comparison of flow artifacts, myocardium edge sharpness, and homogeneity for each sequence obtained from a consensus reading

\begin{tabular}{llll}
\hline & $\begin{array}{l}\text { Flow } \\
\text { artifact }\end{array}$ & $\begin{array}{l}\text { Myocardium } \\
\text { sharpness }\end{array}$ & $\begin{array}{l}\text { Myocardium } \\
\text { homogeneity }\end{array}$ \\
\hline Spiral-FFE & + & ++ & ++ \\
TFE-highres- & ++ & ++ & ++ \\
flowcomp & & & - \\
TFE-highres & - & + & + \\
TFE-lowres & + & - & +
\end{tabular}

The '+' always represents the best image quality for each criteria analyzed (++: best image quality, -: worst image quality). FFE fast field echo; TFE turbo field echo; flowcomp flow compensation; highres high resolution; lowres low resolution

neous signal in the left cavity. Absence of flow artifacts in the left cavity was also observed in the low-resolution TFE sequence and to a lesser extent in the spiral-FFE sequence with flow compensation but without complexconjugate reconstruction. The TFE-highres sequence was degraded by an important flow artifact inside the cavities, as can be seen in Fig. 2. From consensus reading, the best sequence was the Cartesian TFE with both flow compensation and complex-conjugate reconstruction (TFE-highres-flowcomp). Using this sequence, highresolution images with sharp borders between the blood and myocardium were constantly obtained, which was not the case for the other sequences, as demonstrated in Figs. 1, 2, and 3.

Diastolic and systolic volumes as well as the ejection fraction were measured for all MR sequences using the modified Simpson method and by summation of contig- 



Fig. 2 Representation of the flow artifacts from a systole to a diastole (selection of eight phases) for spiral-FFE (a), TFE-highres-flowcomp (b), TFE-highres (c), and TFE-lowres (d). TFE-highres-flowcomp sequence shows a uniform blood signal. The flow-artifact intensity in the spiral sequence is more pronounced in the early diastole. Note also a pronounced artifact in the TFE-highres (c) acquisition. The flow is more homogeneous for the TFE-lowres at the expense of low spatial resolution. FFE fast field echo; TFE turbo field echo; flowcomp flow compensation; highres high resolution; lowres low resolution. Scale bar $=1 \mathrm{~cm}$
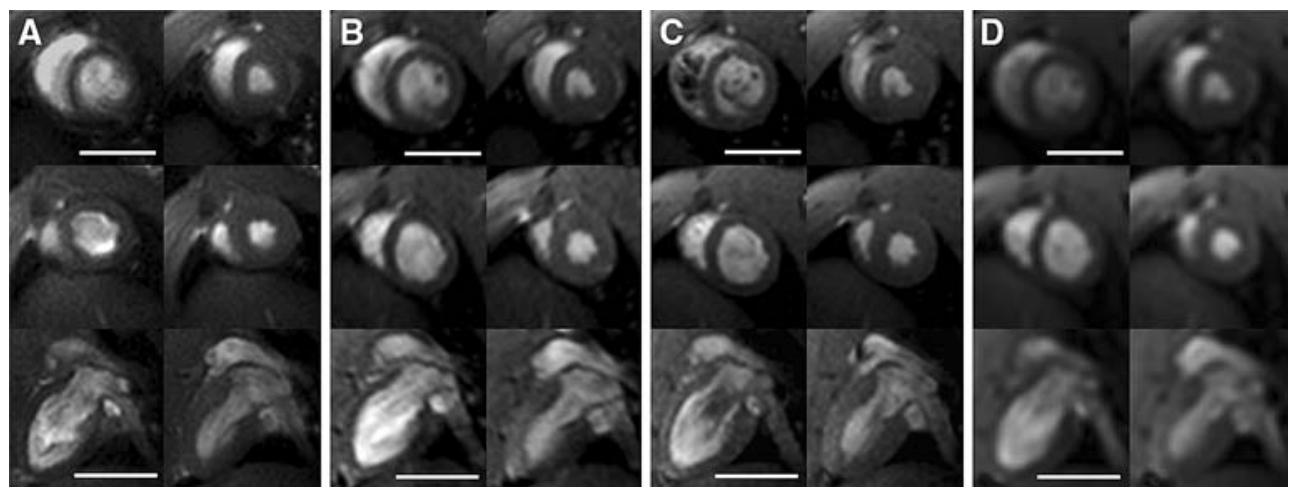

Fig. 3 Rat heart imaged with sequences spiral-FFE (a), TFE-highres-flowcomp (b), TFE-highres (c), and TFE-lowres (d). Diastolic and systolic phases are represented for the short axis (mitral: row 1, and apex: row 2) and the long axis (row 3). The spiral-FFE sequence (a) shows a greater contrast comparing to the TFE-highres-flowcomp (b, c), but the septum is partially delineated due to flow artifact. Even though a good contrast for the TFE-lowres acquisition (d), the sharpness of the image obtained is not satisfactory. Hence the turbo field echo sequences $(\mathbf{b}, \mathbf{c})$ are of great interest. Moreover, the flow compensation for $\mathbf{b}$ decreases dramatically the flux artifacts. FFE fast field echo, TFE turbo field echo, flowcomp flow compensation, highres high resolution, lowres low resolution. Scale bar $=1 \mathrm{~cm}$

uous slices for the TFE-highres-flowcomp sequence. The results are presented in Table 4. No significant differences were found between the four sequences $(p>0.05)$.

For each MRI sequence the left-ventricle mass was compared with the left-ventricle wet weight obtained during autopsy (Table 5). There were no significant differences between the MR-calculated volume and real volume as assessed by autopsy.

From the statistical analysis of the inter- and intraobserver measurements variability was very small. Indeed, the standard errors of mean were between $1.12 \%$ and $4.73 \%$ for intra-observer variability and between
$1.57 \%$ and $3.90 \%$ for inter-observer variability for the sequence chosen.

\section{Discussion}

This work demonstrates that high spatial and temporal resolution images of rat cardiac cine can reliably be obtained on a clinical 1.5-T MR system and can yield accurate cardiac function estimates. Moreover, the acquisition is reproducible. The current in-plane resolution retained in this study was $0.31 \mathrm{~mm}$ after zero-filling $(0.56 \mathrm{~mm}$ ac- 
Table 4 Values of diastolic, systolic volumes and the ejection fraction measured and calculated for each sequence (mean \pm SD)

\begin{tabular}{lcccc}
\hline & Spiral-FFE & $\begin{array}{l}\text { TFE-highres- TFE- } \\
\text { flowcomp }\end{array}$ & $\begin{array}{l}\text { TFE- } \\
\text { highres }\end{array}$ & lowres \\
\hline $\begin{array}{l}\text { Diastolic } \\
\text { volume }(\mu 1)\end{array}$ & $512 \pm 72$ & $569 \pm 72$ & $555 \pm 76$ & $519 \pm 53$ \\
$\begin{array}{l}\text { Systolic } \\
\text { volume }(\mu 1)\end{array}$ & $117 \pm 22$ & $166 \pm 29$ & $151 \pm 23$ & $160 \pm 23$ \\
$\begin{array}{l}\text { Ejection } \\
\text { fraction }(\%)\end{array}$ & $77 \pm 4$ & $71 \pm 4$ & $72 \pm 4$ & $69 \pm 5$ \\
\hline
\end{tabular}

FFE fast field echo; TFE turbo field echo; flowcomp flow compensation; highres high resolution; lowres low resolution

Table 5 Heart mass values in four rats

\begin{tabular}{lc}
\hline & LV mass $(\mathrm{g})$ \\
\hline Spiral-FFE & $0.80 \pm 0.08$ \\
TFE-highres-flowcomp & $0.76 \pm 0.15$ \\
TFE-highres & $0.72 \pm 0.12$ \\
TFE-lowres & $0.63 \pm 0.15$ \\
TFE-flowcomp whole heart & $0.71 \pm 0.18$ \\
Autopsy & $0.82 \pm 0.07$ \\
\hline
\end{tabular}

The weight was evaluated for each MRI sequence and compared with wet weight assessed through autopsy (mean \pm SD). There were no significant differences between the measurements obtained with MRI against real weight (T test). FFE fast field echo; TFE turbo field echo; flowcomp flow compensation; highres high resolution; lowres low resolution; whole heart MRI slices $2 \mathrm{~mm}$ thick along the long heart axis

quired) with a temporal resolution allowing the imaging of 14 phases per heart beat for a heart rate of $360 \mathrm{bpm}$. A similar resolution of $0.5 \mathrm{~mm}$ with $10-14$ phases per heart beat has already been obtained on a clinical 1.5-T MR system in the mouse [18]. However, no detailed indications on the sequence parameters (such as acquisition time, repetition time, echo time, bandwidth, or interpolation) and no validation of the method were provided in this preliminary study. It should be emphasized that in cardiac cine imaging spatial resolution is closely related to temporal resolution. For a fixed acquisition time, spatial resolution can be increased by reducing the number of cardiac phases imaged. For cardiac cine at 1.5-T, higher in-plane resolution of $0.19 \mathrm{~mm}$ in mice $[15,16]$ or $0.35 \mathrm{~mm}$ in the rat [17] have been used. The matrices were obtained at the expense of temporal resolution since the number of cardiac phases of the cine was limited to five or six. To maintain a high number of heart phases, we kept the repetition time as short as possible (7.7 versus $39 \mathrm{~ms}$ in the Franco et al. protocol) by increasing the field of view (FOV) and compensated the loss of SNR by averaging. This strategy yields a high resolution in both the spatial and temporal dimensions while maintaining a reasonably short acquisition time per slice (less than $1.5 \mathrm{~min})$. It should be emphasized that the number of phases could be easily increased if needed by reducing the TFE factor (percentage of matrix acquired within one TR) from three to one or by using the option of phase sharing during the reconstruction $[19,20]$.

A low in-plane resolution (reconstructed: $0.62 \times$ $0.62 \mathrm{~mm}$, acquired: $1.00 \times 1.00 \mathrm{~mm}$ ) was tested to increase the number of phases: 20 phases per cardiac cycle were obtained at a cardiac frequency of $360 \mathrm{bpm}$ with a high CNR. However, the resulting low resolution appears as a limiting factor for myocardium segmentation in comparison to others sequences [21].

The resolution achieved in this study (with a heart diameter of $15 \mathrm{~mm}$ for the rat [6]) has to be compared to values obtained in the human heart which is almost seven times larger in diameter (approximately $100 \mathrm{~mm}$ ) [6]: $2.2 \times 1.4 \mathrm{~mm}^{2}$ in-plane resolution for standard cine sequence and $4.2 \times 2.7 \mathrm{~mm}^{2}$ for real-time cine [22] for human studies and $0.31 \times 0.31 \mathrm{~mm}^{2}$ in this study. Therefore, the spatial resolution in the rat, normalized to heart diameter is between the resolution of the standard cine and the real-time cine.

The typical resolution of high-field MR systems dedicated to small animals is $0.39 \mathrm{~mm}$ with $11-12$ cardiac phases per heart beat at 4.7-T [10], or $0.23 \mathrm{~mm}$ with 12 frames per heart cycle at 7.05-T $[12,13]$. The spatial resolution obtained on our clinical MR scanner without losing temporal resolution is lower than the spatial resolution that can be achieved with small-animal dedicated systems, but yet enables sufficient image quality to accurately delineate heart contours.

Regarding the choice of the MR sequences, in order to suppress flow artifacts, it was important to use flow compensation (gradient moment nulling of the first order). The use of a clinical scanner (i.e., 1.5-T) implies longer gradient rise time compared to high-field systems, hence imposing longer acquisition times. Consequently, the system is more sensitive to flow artifacts which justifies the use of the flow-compensation parameter. The TFEhighres-flowcomp sequence with this correction exhibited a homogeneous blood signal in ventricles, as shown in Fig. 2.

Using a spiral sequence, higher resolution as well as high blood-to-myocardium contrast can be obtained $(p<0.02)$ (Table 2). However, flow artifacts at the blood-myocardium interface were often present in systole (Figs. 2, 3). This result was at first surprising as spiral sequences are known to be more robust to flow artifacts due to the intrinsic gradient moment-nulling of the sequence. In our sequence, the high number of spiral interleaves (256) decreases drastically the number of rotations per spiral. The resulting trajectory covers less than one 
quarter of $k$-space and does no longer have the gradient moment-nulling property.

We did not evaluate balanced steady-state free-precession sequence (True Fisp on Siemens MR scanners) that is one of the reference MR sequences for cardiac cine in patients. In our studies, True Fisp imaging on the rat heart was degraded by severe banding artifacts that could not be resolved by the shim available on our MR system. Flow artifacts that may be related to off-resonance effects [23] were also present.

Due to the high image quality of the TFE-highresflowcomp sequence, the variation of measurements was very small (Table 4 ), and the measurements were comparable to published data [17,24]. The low inter- and intraobserver variability of the heart function measurements demonstrates the reproducibility and feasibility of determining physiological characteristics of the myocardium with a 1.5-T MR system, indicating that this protocol yields robust measurements.

The following physiological values are presented in Tables 4 and 5 and are in agreement with previous studies made at different field strengths (from 1.5 to $7-T$ ): the ejection fraction [10,12,17,25], left-ventricular diastolic [10] and systolic volumes $[10,26]$, and left-ventricular mass $[10,13,25]$.

This study demonstrated an efficient protocol to measure in less than $30 \mathrm{~min}$ the cardiac function of rat on a clinical 1.5-T MR system. In view of the availability of such systems installed in cardiac research centers, smallanimal cardiovascular research may progress rapidly in the near future. However, high-resolution imaging using other cardiac MR sequences (such as tag, perfusion, or late enhancement) needs to be implemented in future work.

\section{Conclusion}

We performed a systematic comparison of fast gradientecho cine sequences in the rat. The best MR sequence was the TFE-highres-flowcomp MR sequence with flow compensation and complex-conjugated reconstruction, yielding high temporal and spatial resolution. As reproducible images of cardiac cine were obtained in the rat, we demonstrated that a clinical 1.5-T MR system successfully monitors cardiac function of small animals.

Acknowledgments We thank the National Swiss Foundation (Grant PPOB-68778), the Leenaards Foundation and the Department of Radiology of the Geneva University Hospital for financial support, as well as A. Cernicanu and J. Diaper for correcting the manuscript for English language.

\section{References}

1. Earls JP, Ho VB, Foo TK, Castillo E, Flamm SD (2002) Cardiac MRI: recent progress and continued challenges. J Magn Reson Imaging 16:111-127

2. Pujadas S, Reddy GP, Weber O, Lee JJ, Higgins CB (2004) MR imaging assessment of cardiac function. J Magn Reson Imaging 19:789-799

3. Gotte MJ, van Rossum AC, Twisk JWR, Kuijer JPA, Marcus JT, Visser CA (2001) Quantification of regional contractile function after infarction: strain analysis superior to wall thickening analysis in discriminating infarct from remote myocardium. J Am Coll Cardiol 37:808-817

4. Sensky PR, Jivan A, Hudson NM, Keal RP, Morgan B, Tranter JL, de Bono D, Samani NJ, Cherryman GR (2000) Coronary artery disease: combined stress MR imaging protocol-one-stop evaluation of myocardial perfusion and function. Radiology 215:608-614

5. Pennell DJ, Sechtem UP, Higgins CB, Manning WJ, Pohost GM,

Rademakers FE, van Rossum AC, Shaw LJ, Yucel EK (2004) Clinical indications for cardiovascular magnetic resonance (CMR): Consensus Panel report. Eur Heart J 25:1940-1965
6. Brau AC, Hedlund LW, Johnson GA (2004) Cine magnetic resonance microscopy of the rat heart using cardiorespiratory-synchronous projection reconstruction. J Magn Reson Imaging 20:31-38

7. Saeed M, Watzinger N, Krombach GA, Lund GK, Wendland MF, Chujo M, Higgins CB (2002) Left ventricular remodeling after infarction: sequential MR imaging with oral nicorandil therapy in rat model. Radiology 224:830-837

8. Yang Z, Berr SS, Gilson WD, Toufektsian MC, French BA (2004) Simultaneous evaluation of infarct size and cardiac function in intact mice by contrast-enhanced cardiac magnetic resonance imaging reveals contractile dysfunction in noninfarcted regions early after myocardial infarction. Circulation 109:1161-1167

9. Williams SP, Gerber HP, Giordano FJ, Peale FVJr. Bernstein LJ, Bunting S, Chien KR, Ferrara N, van Bruggen N (2001) Dobutamine stress cine-MRI of cardiac function in the hearts of adult cardiomyocyte-specific VEGF knockout mice. J Magn Reson Imaging 14:374-382
10. Thomas D, Ferrari VA, Janik M, Kim DH, Pickup S, Glickson JD, Zhou R (2004) Quantitative assessment of regional myocardial function in a rat model of myocardial infarction using tagged MRI. Magn Reson Mater Phys 17:179-187

11. Epstein FH, Yang Z, Gilson WD, Berr SS, Kramer CM, French BA (2002) MR tagging early after myocardial infarction in mice demonstrates contractile dysfunction in adjacent and remote regions. Magn Reson Med 48:399-403

12. Nahrendorf M, Wiesmann F, Hiller $\mathrm{KH}, \mathrm{Hu} \mathrm{K}$, Waller C, Ruff J, Lanz TE, Neubauer S, Haase A, Ertl G, Bauer WR (2001) Serial cine-magnetic resonance imaging of left ventricular remodeling after myocardial infarction in rats. J Magn Reson Imaging 14:547-555 
13. Nahrendorf M, Hiller KH, Greiser A, Kohler S, Neuberger T, Hu K, Waller C, Albrecht M, Neubauer S, Haase A, Ertl G, Bauer WR (2003) Chronic coronary artery stenosis induces impaired function of remote myocardium: MRI and spectroscopy study in rat. Am J Physiol Heart Circ Physiol 285:H2712-H2721

14. Chapon C, Franconi F, Lemaire L, Marescaux L, Legras P, Saint-Andre JP, Denizot B, Le Jeune JJ (2003) High field magnetic resonance imaging evaluation of superparamagnetic iron oxide nanoparticles in a permanent rat myocardial infarction. Invest Radiol 38:141-146

15. Franco F, Dubois SK, Peshock RM, Shohet RV (1998) Magnetic resonance imaging accurately estimates LV mass in a transgenic mouse model of cardiac hypertrophy. Am J Physiol 274:H679-H683

16. Franco F, Thomas GD, Giroir B, Bryant D, Bullock MC, Chwialkowski MC, Victor RG, Peshock RM (1999) Magnetic resonance imaging and invasive evaluation of development of heart failure in transgenic mice with myocardial expression of tumor necrosis factor-alpha. Circulation 99:448-454
17. Jones JR, Mata JF, Yang Z, French BA, Oshinski JN (2002) Left ventricular remodeling subsequent to reperfused myocardial infarction: evaluation of a rat model using cardiac magnetic resonance imaging. J Cardiovasc Magn Reson 4:317-326

18. Oh YS, Thomson LE, Fishbein MC, Berman DS, Sharifi B, Chen PS (2004) Scar formation after ischemic myocardial injury in MRL mice. Cardiovasc Pathol 13:203-206

19. Foo TK, Bernstein MA, Aisen AM, Hernandez RJ, Collick BD, Bernstein T (1995) Improved ejection fraction and flow velocity estimates with use of view sharing and uniform repetition time excitation with fast cardiac techniques. Radiology 195:471-478

20. Markl M, Schneider B, Hennig J (2002) Fast phase contrast cardiac magnetic resonance imaging: improved assessment and analysis of left ventricular wall motion. J Magn Reson Imaging 15:642-653

21. Roussakis A, Baras P, Seimenis I, Andreou J, Danias PG (2004) Relationship of number of phases per cardiac cycle and accuracy of measurement of left ventricular volumes, ejection fraction, and mass. J Cardiovasc Magn Reson 6:837-844
22. Lee VS, Resnick D, Bundy JM, Simonetti OP, Lee P, Weinreb JC (2002) Cardiac function: MR evaluation in one breath hold with real-time true fast imaging with steady-state precession. Radiology 222:835-842

23. Storey P, Li W, Chen Q, Edelman RR (2004) Flow artifacts in steady-state free precession cine imaging. Magn Reson Med 51:115-122

24. Plein S, Bloomer TN, Ridgway JP Jones TR, Bainbridge GJ, Sivananthan MU (2001) Steady-state free precession magnetic resonance imaging of the heart: comparison with segmented $\mathrm{k}$-space gradient-echo imaging. J Magn Reson Imaging 14:230-236

25. Nahrendorf M, Hu K, Fraccarollo D, Hiller KH, Haase A, Bauer WR, Ertl G (2003) Time course of right ventricular remodeling in rats with experimental myocardial infarction. Am J Physiol Heart Circ Physiol 284:H241-H248

26. Wise RG, Huang CL, Gresham GA, Al-Shafei AI, Carpenter TA, Hall LD (1998) Magnetic resonance imaging analysis of left ventricular function in normal and spontaneously hypertensive rats. J Physiol 513(Pt. 3):873-887 